Supplementary Information

\title{
Influence of Dioxygen on the Promotional Effect of Bi during Pt-catalyzed Oxidation of 1,6-Hexanediol
}

\author{
Jiahan Xie ${ }^{\mathrm{a}}$, Benjamin Huang ${ }^{\mathrm{a}}$, Kehua Yin ${ }^{\mathrm{a}}$, Hien N. Pham ${ }^{\mathrm{b}}$, \\ Raymond R. Unocic ${ }^{c}$, Abhaya K. Datye ${ }^{\mathrm{b}}$, Robert J. Davis ${ }^{\mathrm{a}}{ }^{*}$ \\ Department of Chemical Engineering, University of Virginia, 102 Engineers’ Way, PO Box \\ 400741, Charlottesville, VA 22904-4741, United States
}

a: Department of Chemical Engineering, Charlottesville, University of Virginia, VA 22904-4741, United States

b: Department of Chemical Engineering and Center for Microengineered Materials, University of New Mexico, Albuquerque, NM 87131, United States

c: Center for Nanophase Materials Sciences, Oak Ridge National Laboratory, Oak Ridge, TN 37831, United States

*Author to whom correspondence is addressed

Phone: 1 - 434 - 924 - 6284

Email: rjd4f@virginia.edu 


\section{Calculation of Weisz-Prater parameter under different conditions}

Based on the Weisz-Prater criterion, the diffusion limitations are negligible when $\Phi_{i}=\frac{r^{e f f} \cdot r_{p}^{2}}{c_{i, s} \cdot D_{i, m i x}^{e f f}}<0.3$

with $\Phi_{i}$, the dimensionless Weisz-Prater parameter; $r^{\text {eff }}$, effective (observed) rate per volume of catalyst in mol s $\mathrm{m}^{-1} ; r_{p}$, radium of the catalyst particle in $\mathrm{m} ; c_{i, s}$, concentration of $\mathrm{i}$ at the surface of the particle in mol $\mathrm{m}^{-3}$ and $D_{i, m i x}$ eff, effective diffusivity of $i$ in the mixture in $\mathrm{m}^{2} \mathrm{~s}^{-1}$

The Weisz-Prater parameter of $\mathrm{O}_{2}$ was calculated under $0.02 \mathrm{MPa} \mathrm{O}_{2}$ over $\mathrm{Pt} / \mathrm{C}$ with different HDO concentration $(0.05-0.2 \mathrm{M})$ and temperature $(318-338 \mathrm{~K})$. The $r^{\text {eff }}$ was measured as the initial reaction rate (Table S3). The $r_{p}$ was determined from the SEM image, which was estimated to be $4 \mu \mathrm{m}$ as $1 / 6$ of the particle diameter ${ }^{1}$ (Figure S1). The $c_{i, s}$ was assumed to be the bulk concentration, $c_{i, l}$. The bulk concentration of HDO was used as the initial concentration and for $\mathrm{O}_{2}$, Henry's law was applied ${ }^{2}$ :

$P_{O_{2}}=H_{O_{2}, \text { mix }} \cdot \frac{c_{O_{2}, l}}{c_{\text {total }}}$

with $P_{\mathrm{O}_{2}}$, saturated vapor pressure of oxygen in $\mathrm{Pa} ; H_{\mathrm{O}_{2}, \text { mix }}$, Henry coefficient of oxygen in the liquid mixture in $\mathrm{Pa}$ and $c_{\text {total }}$ as the total concentration of liquid, which is $5.10 \times 10^{3}$ mol m${ }^{-3} \cdot$ As the concentration of HDO is low $(0.1 \mathrm{M}), H_{O_{2}}$ mix was assumed to be equal to $\mathrm{H}_{\mathrm{O}_{2}, \mathrm{H}_{2} \mathrm{O}}$, which was calculated as a function of temperature according to a correlation of Rettich et al. ${ }^{4}$. The effective diffusivity, $D_{i, m i x}^{e f f}$, was calculated by

$D_{i, m i x}^{e f f}=D_{i, m i x} \cdot \frac{\varepsilon}{\tau}$ 
where $\varepsilon$ and $\tau$ are the catalyst porosity and tortuosity, respectively, which were estimated to be 0.4 and 5 , as a first approximation. The diffusion coefficient of dioxygen, $D_{O_{2} \text {,mix }}$, was

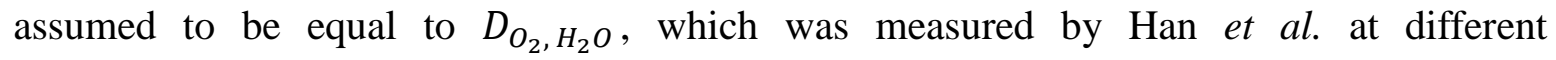
temperatures. $^{5}$

Based on Equations 1 - 3, the Weisz-Prater parameters of dioxygen, shown in Table S3, were calculated under different conditions and were all below 0.3 . 
Tables and Figures

Table S1. XPS curve fitting parameters used in Casa XPS(Version 2.3.16)

\begin{tabular}{cccc}
\hline Species & $\operatorname{Pt}(0)$ & $\operatorname{Bi}(0)$ & $\operatorname{Bi}(\mathrm{III})$ \\
\hline Position $(\mathrm{eV})$ & $70.3-71.3$ & $156.5-157.8$ & $158.8-160.0$ \\
FWHM & $0.3-1.5$ & $0.2-1.2$ & $1-2.5$ \\
Line shape & $\mathrm{LA}(1.2,85,70)$ & $\mathrm{LA}(1.2,85,70)$ & $\mathrm{GL}(30)$ \\
\hline
\end{tabular}


Table S2. Elemental compositions from EDS of $0.6 \mathrm{Bi}-\mathrm{Pt} / \mathrm{C}, 0.6 \mathrm{Bi}-\mathrm{Pt} / \mathrm{C}-\mathrm{P}$ and $0.6 \mathrm{Bi}-\mathrm{Pt} / \mathrm{C}-\mathrm{R}^{\mathrm{a}}$.

\begin{tabular}{|c|c|c|c|c|c|c|c|c|}
\hline \multicolumn{3}{|c|}{$0.6 \mathrm{Bi}-\mathrm{Pt} / \mathrm{C}$} & \multicolumn{3}{|c|}{$0.6 \mathrm{Bi}-\mathrm{Pt} / \mathrm{C}-\mathrm{P}^{\mathrm{b}}$} & \multicolumn{3}{|c|}{$0.6 \mathrm{Bi}-\mathrm{Pt} / \mathrm{C}-\mathrm{R}^{\mathrm{b}}$} \\
\hline Region & $\begin{array}{c}\text { Pt } \\
(\mathrm{mol} \%)\end{array}$ & $\begin{array}{c}\mathrm{Bi} \\
(\mathrm{mol} \%)\end{array}$ & Region & $\begin{array}{c}\text { Pt } \\
(\mathrm{mol} \%)\end{array}$ & $\begin{array}{c}\mathrm{Bi} \\
(\mathrm{mol} \%)\end{array}$ & Region & $\begin{array}{c}\mathrm{Pt} \\
(\mathrm{mol} \%)\end{array}$ & $\begin{array}{c}\mathrm{Bi} \\
(\mathrm{mol} \mathrm{\%})\end{array}$ \\
\hline a1 & 60 & 40 & $\mathrm{c} 1$ & 80 & 20 & e1 & 75 & 25 \\
\hline a2 & 74 & 26 & c2 & 93 & 7 & e2 & 76 & 24 \\
\hline a3 & 97 & 3 & c3 & 84 & 16 & e3 & 55 & 45 \\
\hline $\mathrm{a} 4$ & 83 & 17 & c4 & 61 & 39 & e4 & 82 & 18 \\
\hline a5 & 60 & 40 & c5 & 64 & 36 & e5 & 61 & 39 \\
\hline a6 & 69 & 31 & c6 & 80 & 20 & e6 & 49 & 51 \\
\hline a7 & 83 & 17 & C7 & 52 & 48 & e7 & 0 & 100 \\
\hline a8 & 50 & 50 & C8 & 60 & 40 & e8 & 79 & 21 \\
\hline a9 & 87 & 13 & c9 & 61 & 39 & e9 & 25 & 75 \\
\hline a10 & 88 & 12 & c10 & 59 & 41 & e10 & 76 & 24 \\
\hline b1 & 62 & 38 & $\mathrm{~d} 1$ & 79 & 21 & f1 & 0 & 100 \\
\hline b2 & 50 & 50 & $\mathrm{~d} 2$ & 79 & 21 & $\mathrm{f} 2$ & 0 & 100 \\
\hline b3 & 90 & 10 & d3 & 79 & 21 & f3 & 38 & 62 \\
\hline b4 & 75 & 25 & $\mathrm{~d} 4$ & 57 & 44 & $\mathrm{f} 4$ & 0 & 100 \\
\hline b5 & 69 & 31 & d5 & 85 & 15 & f5 & 43 & 57 \\
\hline b6 & 55 & 45 & d6 & 79 & 21 & f6 & 11 & 89 \\
\hline b7 & 52 & 38 & d7 & 75 & 25 & f7 & 49 & 51 \\
\hline b8 & 60 & 40 & d8 & 86 & 14 & f8 & 68 & 32 \\
\hline b9 & 70 & 30 & d9 & 69 & 31 & f9 & 59 & 41 \\
\hline b10 & 100 & 0 & $\mathrm{~d} 10$ & 83 & 17 & $\mathrm{f} 10$ & 46 & 54 \\
\hline Avg & $72 \pm 15$ & $28 \pm 15$ & Avg & $73 \pm 12$ & $27 \pm 12$ & Avg & $45 \pm 30$ & $55 \pm 30$ \\
\hline
\end{tabular}

a: The corresponding regions for EDS analysis are shown in Figure S7.

b: Catalyst collected after 20 min of pretreatment in 0.1 M 1,6-hexnaediol under He flow at $328 \mathrm{~K}$.

c: Catalyst collected after $4 \mathrm{~h}$ of 1,6-hexanediol oxidation under $0.02 \mathrm{MPa}_{2}$ at $328 \mathrm{~K}$. 
Table S3. Calculated Weisz-Prater parameters for dioxygen

\begin{tabular}{cccccccc}
\hline $\mathrm{c}(\mathrm{HDO})$ & $\mathrm{P}\left(\mathrm{O}_{2}\right)$ & $\mathrm{T}$ & $r^{\mathrm{eff}}$ & $\mathrm{H}_{\mathrm{O}_{2}, \mathrm{H}_{2} \mathrm{O}^{4}}$ & $C_{O_{2}}$ & $D_{O_{2}}{ }^{5}$ & $\Phi_{O_{2}}$ \\
$\left(\mathrm{~mol} \mathrm{~L}^{-1}\right)$ & $(\mathrm{MPa})$ & $(\mathrm{K})$ & $\left(\mathrm{mol} \mathrm{s}^{-1} \mathrm{~m}^{-3}\right)$ & $\left(10^{9} \mathrm{~Pa}\right)$ & $\left(\mathrm{mol} \mathrm{m}^{-3}\right)$ & $\left(10^{-9} \mathrm{~m}^{2} \mathrm{~s}^{-1}\right)$ & \\
\hline 0.05 & 0.02 & 328 & 0.84 & 6.19 & 0.16 & 3.64 & 0.180 \\
0.1 & 0.02 & 328 & 0.95 & 6.19 & 0.16 & 3.64 & 0.203 \\
0.15 & 0.02 & 328 & 1.18 & 6.19 & 0.16 & 3.64 & 0.253 \\
0.1 & 0.02 & 318 & 0.72 & 5.68 & 0.18 & 3.05 & 0.168 \\
0.1 & 0.02 & 338 & 1.18 & 6.7 & 0.15 & 4.25 & 0.234 \\
\hline
\end{tabular}



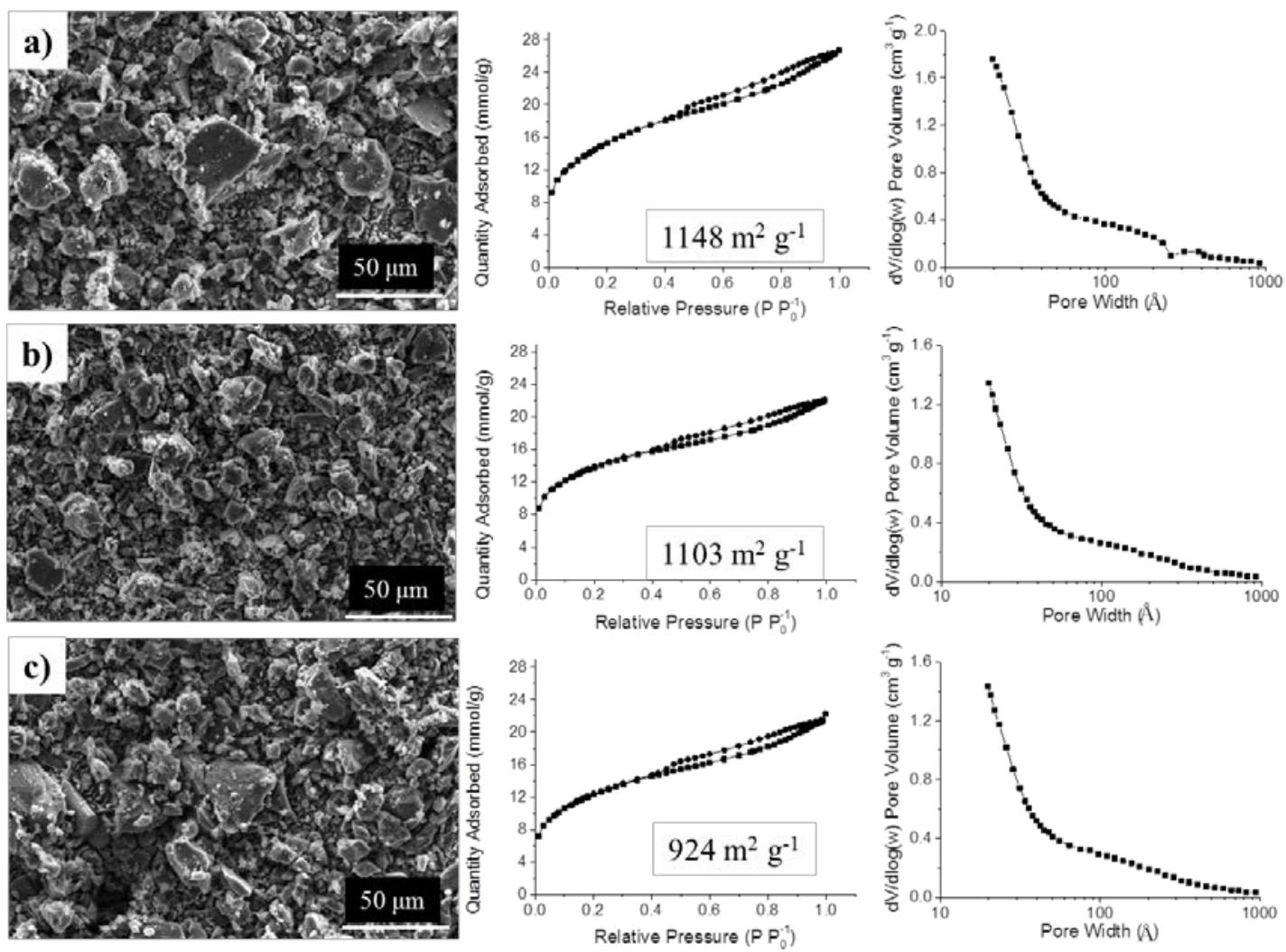

Figure S1. SEM images, $\mathrm{N}_{2}$ sorption isotherm, measured surface areas measured and pore size distribution curves of a) $\mathrm{Pt} / \mathrm{C}$ as received; b) $0.6 \mathrm{Bi}-\mathrm{Pt} / \mathrm{C}$ as-prepared and c) 0.6Bi-Pt/C-R recovered after $4 \mathrm{~h}$ of 1,6-hexanediol oxidation under $0.02 \mathrm{MPa}_{2}$ at $328 \mathrm{~K}$. 

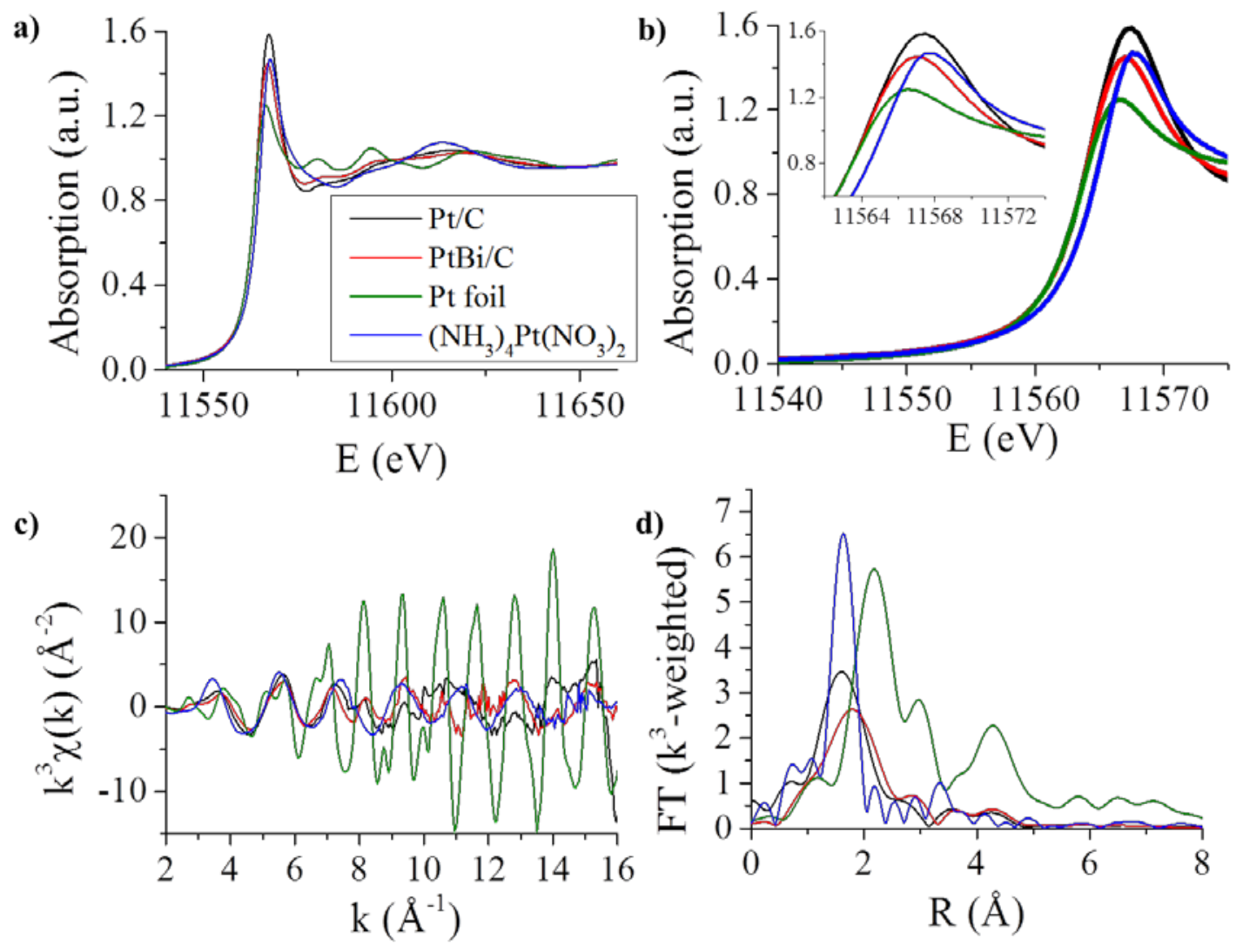

Figure S2. Normalized XAS spectra at the Pt L III edge (a), Normalized XANES spectra at the Pt $\mathrm{L}_{\text {III }}$ edge (b), $k^{3}$-weighted EXAFS data in $k$-space (c) and $r$-space (d) collected during exposure of Pt/C (black), 0.3Bi-Pt/C (red), Pt foil (green) and $\left(\mathrm{NH}_{3}\right)_{4} \mathrm{Pt}\left(\mathrm{NO}_{3}\right)_{2}$ (blue) to air. 

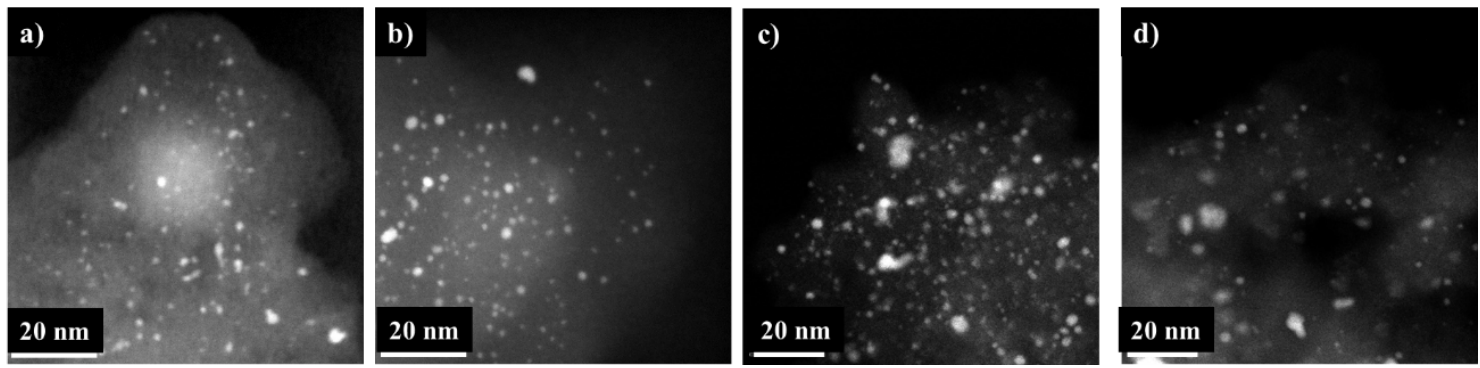

Figure S3. Dark-field STEM images of a) Pt/C, b) $0.3 \mathrm{Bi}-\mathrm{Pt} / \mathrm{C}$, d) $0.6 \mathrm{Bi}-\mathrm{Pt} / \mathrm{C}$ and d) $0.6 \mathrm{Bi}-\mathrm{Pt} / \mathrm{C}-\mathrm{R}$ recovered after $4 \mathrm{~h}$ of 1,6-hexanediol oxidation under $0.02 \mathrm{MPa} \mathrm{O}_{2}$ at $328 \mathrm{~K}$.
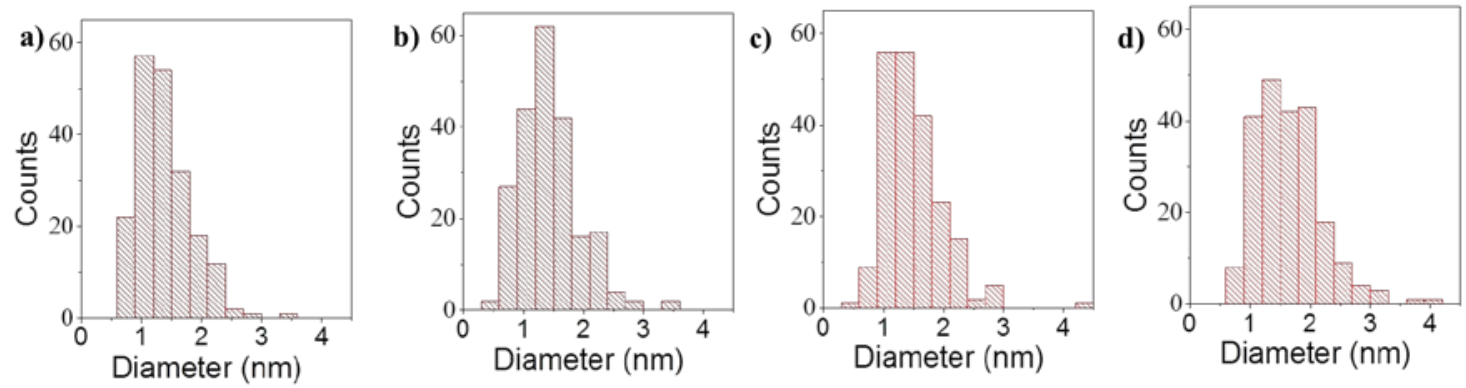

Figure S4. Particle size distributions obtained from STEM of a) Pt/C, b) 0.3Bi-Pt/C, c) $0.6 \mathrm{Bi}-\mathrm{Pt} / \mathrm{C}$ and d) $0.6 \mathrm{Bi}-\mathrm{Pt} / \mathrm{C}$-R recovered after $4 \mathrm{~h}$ of 1,6-hexanediol oxidation under 0.02 $\mathrm{MPa} \mathrm{O}_{2}$ at $328 \mathrm{~K}$. 


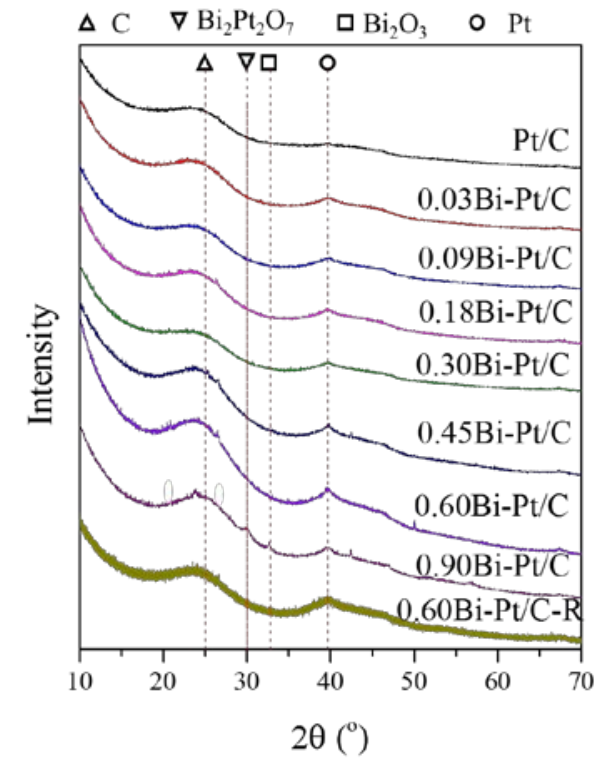

Figure S5. XRD patterns, offset for clarity, of Pt/C and Bi-Pt/C bimetallic catalysts. 


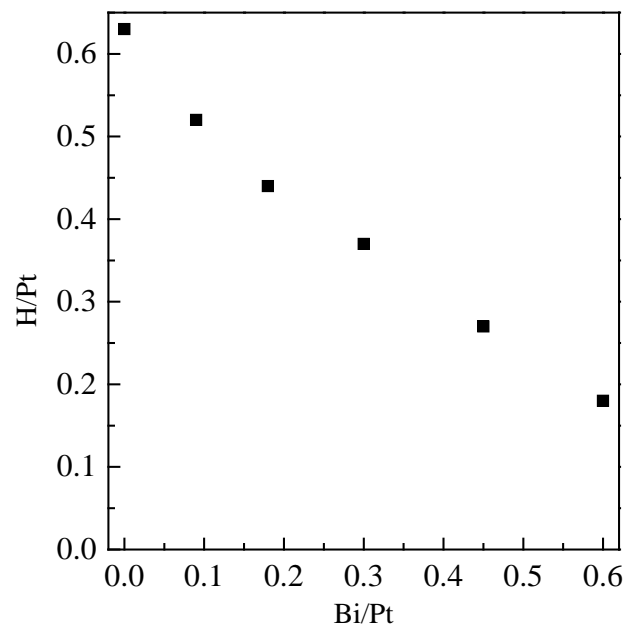

Figure S6. H/Pt ratio of $x \mathrm{Bi}-\mathrm{Pt} / \mathrm{C}$ catalyst $(x=0,0.09,0.18,0.30,0.45,0.6)$. 
a)

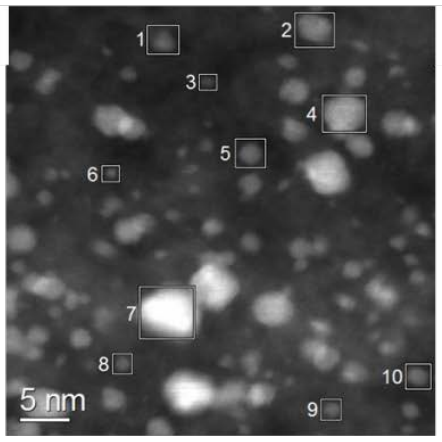

b)

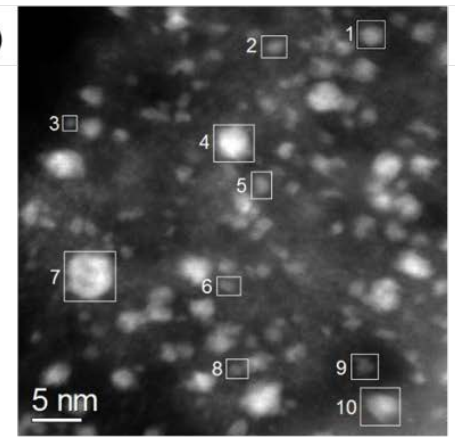

c)

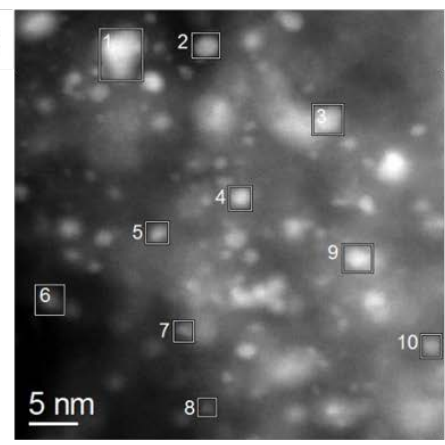

d)

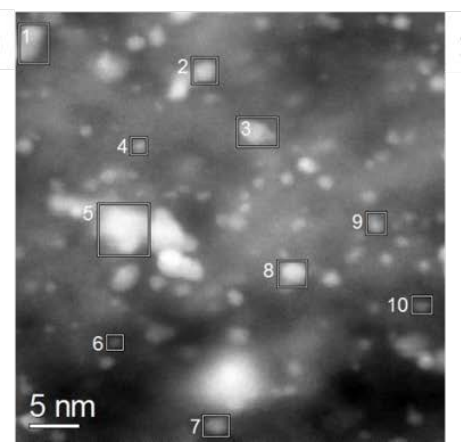

e)

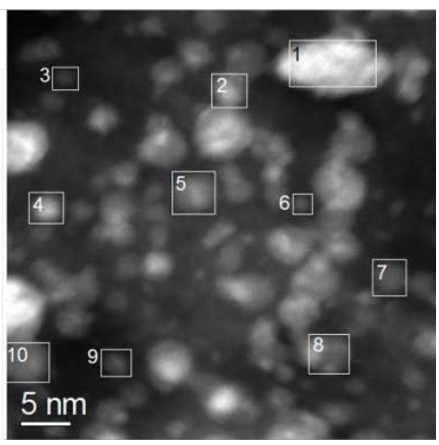

f)

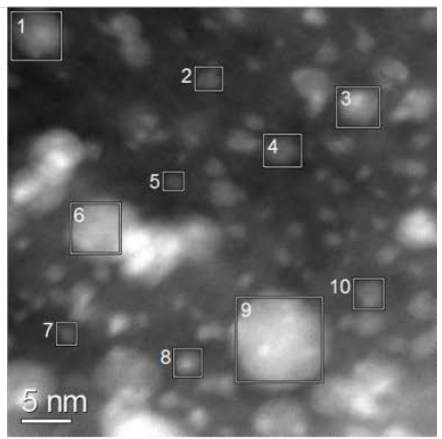

Figure S7. Regions for EDS analysis of a) b) 0.6Bi-Pt/C, c) d) $0.6 \mathrm{Bi}-\mathrm{Pt} / \mathrm{C}-\mathrm{P}$ and e) f) 0.6Bi-Pt/C-R. 0.6Bi-Pt/C-P was recovered after $20 \mathrm{~min}$ pretreatment in $0.1 \mathrm{M}$ hexanediol under He flow at $328 \mathrm{~K}$. 0.6Bi-Pt/C-R was recovered after 4 h reaction of HDO oxidation under $0.02 \mathrm{MPa}_{2}$ at $328 \mathrm{~K}$. The corresponding compositions of analysis region are shown in Table S2. 


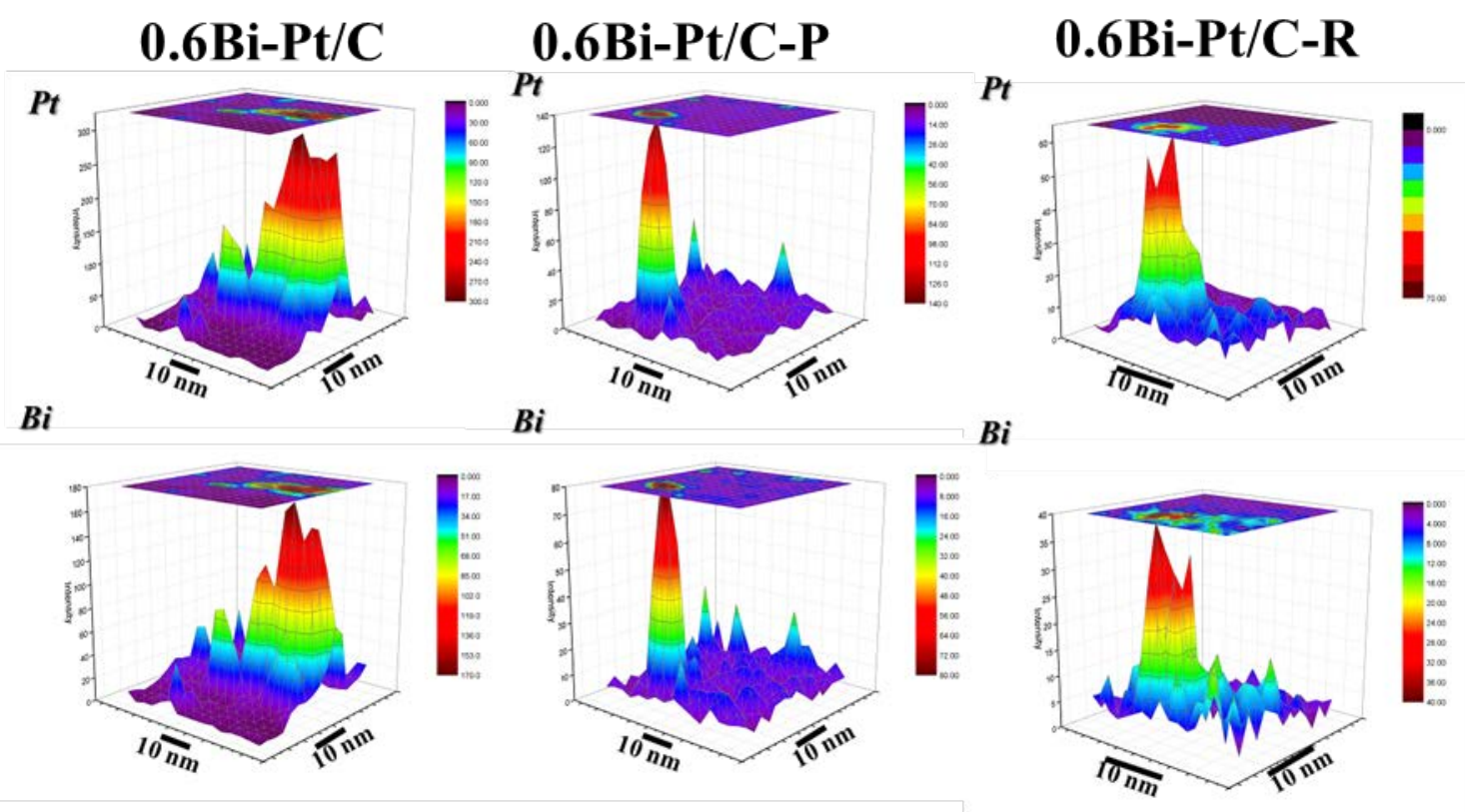

Figure S8. Pt and Bi EDS mapping of 0.6Bi-Pt/C, 0.6Bi-Pt/C-P and 0.6Bi-Pt/C-R in large region. 0.6Bi-Pt/C-P was recovered after 20 min pretreatment in $0.1 \mathrm{M}$ hexanediol under He flow at $328 \mathrm{~K}$. 0.6Bi-Pt/C-R was recovered after $4 \mathrm{~h}$ reaction of HDO oxidation under $0.02 \mathrm{MPa}_{2}$ at $328 \mathrm{~K}$. 

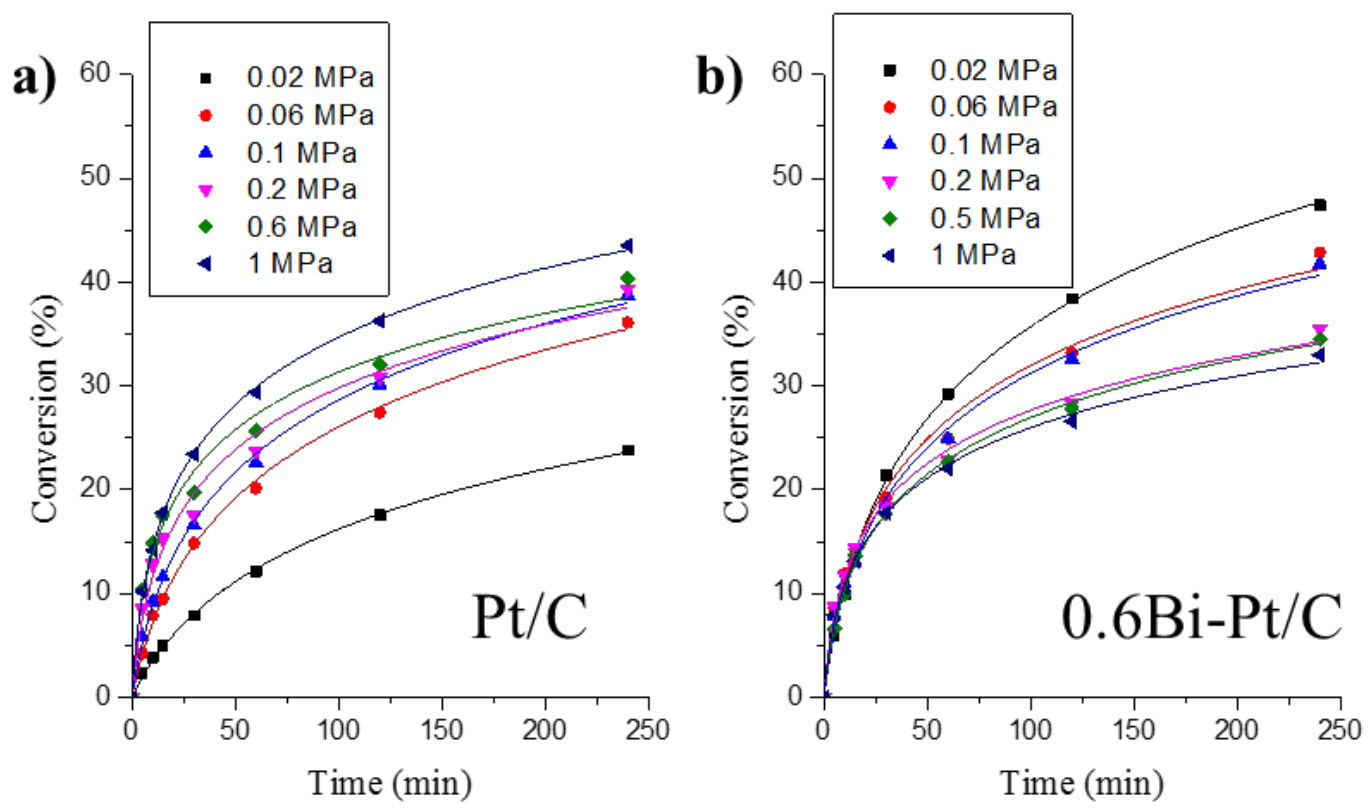

Figure S9. Curve fitting of HDO oxidation deactivation model over $\mathrm{Pt} / \mathrm{C}$ and $0.6 \mathrm{Bi}-\mathrm{Pt} / \mathrm{C}$ under different $\mathrm{O}_{2}$ pressure (dot: measured data; line: fitted curve). 


\section{References:}

(1) Díaz, E.; Casas, J. A.; Mohedano, A. F.; Calvo, L.; Gilarranz, M. A.; Rodríguez, J. J. Ind. Eng. Chem. Res. 2009, 48 (7), 3351-3358.

(2) Henry, W. Phil. Trans. R. Soc. Lond. 1803, 93, 29-274.

(3) Wörz, N.; Brandner, A.; Claus, P. J. Phys. Chem. C 2010, 114, 1164-1172.

(4) Rettich, T. R.; Battino, R.; Wilhelm, E. J. Chem. Thermodyn. 2000, 32 (9), 11451156.

(5) Han, P.; Bartels, D. M. J. Phys. Chem. 1996, 100 (13), 5597-5602. 